\title{
Neuroprotective role of icariin in experimental spinal cord injury via its antioxidant, anti-neuroinflammatory and anti-apoptotic properties
}

\author{
GUIZHI JIA $^{1,2}$, YUQIANG ZHANG ${ }^{3}$, WEIHONG LI $^{1}$ and HONGLIANG DAI ${ }^{4}$ \\ ${ }^{1}$ Department of Physiology, Jinzhou Medical University, Jinzhou, Liaoning 121001; ${ }^{2}$ Department of Physiology, \\ Shanxi Medical University, Taiyuan, Shanxi 030001; ${ }^{3}$ Department of Orthopedics, First Affiliated Hospital; \\ ${ }^{4}$ School of Nursing, Jinzhou Medical University, Jinzhou, Liaoning 121001, P.R. China
}

Received December 14, 2018; Accepted July 12, 2019

DOI: $10.3892 / \mathrm{mmr} .2019 .10537$

\begin{abstract}
Icariin is a type of flavonoid derived from the Chinese herbal plant Epimedium sagittatum Maxim. Mounting evidence has confirmed the beneficial effects of icariin in neurological diseases, including spinal cord injury (SCI). The aim of the present study was to investigate the neuroprotective effects of icariin in SCI and the precise underlying mechanism. The weight-drop injury technique was applied to construct an SCI model in Sprague-Dawley rats. Icariin $(35 \mu \mathrm{mol} / \mathrm{kg})$ was administered orally once daily for 7 consecutive days to examine its neuroprotective effects. The Basso, Beattie and Bresnahan scoring system was used for neurobehavioral evaluation. The water content of the injured spinal cord was measured via the dry-wet weight method. Biochemical indices were examined by colorimetric assay using commercially available kits. Western blot analysis was used to detect protein expression. Icariin significantly accelerated the recovery of the locomotor function of SCI rats and decreased spinal cord water content. Icariin also attenuated SCI-induced pro-apoptotic protein expression and activity, while it increased anti-apoptotic protein levels. In addition, icariin alleviated oxidative stress in SCI rats and decreased the levels of inflammatory molecules, including interleukin (IL)-1 $\beta$, IL-6, tumor necrosis factor- $\alpha$, nitric oxide, nuclear factor- $\mathrm{\kappa} \mathrm{B}$ and inducible nitric oxide synthase, and increased the expression of anti-inflammatory proteins, including NADPH-quinone oxidoreductase-1, heme oxygenase-1 and nuclear factor erythroid 2-related factor 2 in the injured spinal cord. Therefore, icariin treatment accelerated locomotor function recovery in SCI, and its protective
\end{abstract}

Correspondence to: Dr Hongliang Dai, School of Nursing, Jinzhou Medical University, No. 40, Section 3, Songpo Road, Jinzhou, Liaoning 121001, P.R. China

E-mail: jy2006hldai@sohu.com

Key words: icariin, spinal cord injury, inflammation, oxidative stress, apoptosis effects may be mediated via its antioxidant, anti-inflammatory and anti-apoptotic bioactivity.

\section{Introduction}

Spinal cord injury (SCI) is among the most devastating conditions to the health and quality of life of patients and their families $(1,2)$. SCI generally causes severe and permanent neurological deficits, is associated with high healthcare costs, and is becoming a major clinical concern worldwide (3). The pathological events of SCI include primary and secondary injury (4-6). Although both types of injury are responsible for the progressive neuronal death following trauma, there is currently no effective medical or surgical treatment for primary injury, and all efforts are focused on alleviating secondary injury $(6,7)$.

Although the precise pathogenesis of SCI remains elusive, multiple mechanisms, such as inflammation, oxidative stress, immune neurotoxicity, apoptosis and autophagy, have been implicated (8-10). For example, as the currently standard treatment for acute SCI, methylprednisolone exerts neuroprotective effects through its potent anti-inflammatory, anti-apoptotic and antioxidant properties (11). However, the effectiveness of methylprednisolone regarding functional improvement after SCI remains a subject of debate (12); thus, a number of other agents have been trialed for SCI treatment. Recently, Tohda and Nagata (12) confirmed the beneficial effect of icariin, a flavonoid derived from the Chinese herbal plant Epimedium sagittatum Maxim, on experimental SCI based on locomotor rating scale scoring. The underlying protective mechanism, however, remains largely unknown. In order to achieve a better understanding of the beneficial effect of icariin on SCI, the present study attempted to determine its precise underlying mechanism on the basis of previous reports. Attention was specifically focused on the modulatory effect of icariin on apoptotic, redox and inflammatory molecules.

\section{Materials and methods}

Animals and drug treatment. A total of 24 adult male Sprague-Dawley rats (6 weeks old) weighing 180-220 g 
were purchased from the Laboratory Animal Center of Jinzhou Medical University, and were housed in a temperature-controlled room $\left(23 \pm 0.5^{\circ} \mathrm{C}\right)$ with $35-55 \%$ relative humidity and a 12/12-h light/dark cycle. The rats were given free access to food and water throughout the experimental period. All procedures were approved by the Experimental Animal Care and Use Committee at Jinzhou Medical University.

The animals were randomly divided into three groups ( $\mathrm{n}=8$ /group): The sham group, which underwent laminectomy; the SCI group, in which SCI was induced through the Allen method (13), followed by oral administration of $0.1 \%$ $\mathrm{DMSO} /$ saline solution $1 \mathrm{~h}$ after SCI and then once daily for 7 consecutive days; and the icariin treatment group, with oral administration of $35 \mu \mathrm{mol} / \mathrm{kg}$ icariin (Sigma-Aldrich; Merck $\mathrm{KGaA}) 1 \mathrm{~h}$ after SCI and then once daily for 7 consecutive days. Icariin was dissolved in $0.1 \% \mathrm{DMSO} /$ saline solution. The icariin dose used in the present study was determined based on a previous study by Tohda and Nagata (12); in that study, icariin effectively ameliorated SCI-induced motor dysfunction at a $50 \mu \mathrm{mol} / \mathrm{kg}$ dose in mice, which is equivalent to a $35 \mu \mathrm{mol} / \mathrm{kg}$ dose in rats as used in the present study, according to standard dose conversion between mice and rats (the conversion coefficient between mice and rats is 0.7 ). As for the duration of medication therapy, the study by Tohda and Nagata (12) showed that 3 consecutive days' administration of icariin to the SCI mice produced a marked protective effect. The duration was increased to 7 days in the present study in order to ensure that the effectiveness of the agent could be seen.

Establishment of the SCImodel. The SCI model was established as described previously (14). In brief, rats were anesthetized intraperitoneally with $40 \mathrm{mg} / \mathrm{kg}$ sodium pentobarbital and the spinal cord was exposed under aseptic conditions after laminectomy at T9/10. Subsequently, an impactor of $2 \mathrm{~mm}$ diameter and weighing $10 \mathrm{~g}$ was dropped from a height of $25 \mathrm{~mm}$ onto the surface of T9/10, causing spinal cord congestion. In the sham group, only laminectomy was performed.

Locomotion recovery assessment. The locomotion recovery of all rats was assessed using the Basso, Beattie and Bresnahan (BBB) open-field locomotor rating scale, as described previously (7). Two independent examiners who were blinded to the grouping assessed the scores of each group. To ensure there was no baseline discrepancy, BBB scoring was also performed prior to the surgery. The BBB rating scale was scored $0-21$, with higher scores indicating better locomotor function.

Determination of spinal cord water content. Following treatment with icariin for 7 days, the water content of the spinal cord was evaluated. All rats were sacrificed by lethal intraperitoneal injection of $180 \mathrm{mg} / \mathrm{kg}$ sodium pentobarbital, and the spinal cord samples were removed, placed on ice and then dried for $48 \mathrm{~h}$ at $80^{\circ} \mathrm{C}$ for the determination of the dry weights. The water content of the spinal cord was calculated as follows: [(wet weight-dry weight)/wet weight]x100\%.

Western blot analysis. The rats were anesthetized on day 7 after SCI, and the damaged spinal cord was removed around the injury epicenter ( $5 \mathrm{~mm}$ cephalad and caudally). The tissues were lysed in RIPA buffer containing 1\% PMSF (Beyotime
Institute of Biotechnology). The protein content was determined using a bicinchoninic acid kit (Beyotime Institute of Biotechnology). Western blotting was performed as described previously (15). The antibodies used were as follows: Anti-Bax (rabbit polyclonal antibody; 1:500 dilution; cat. no. ab53154), anti-Bcl-2 (rabbit polyclonal antibody; 1:1,000 dilution; cat. no. ab196495), anti-cleaved caspase 3 (rabbit polyclonal antibody; 1:1,000 dilution; cat. no. ab49822), anti-cleaved caspase 9 (rabbit polyclonal antibody; 1:500 dilution; cat. no. ab25758), anti-nuclear factor erythroid 2-related factor 2 (Nrf2; rabbit polyclonal antibody; 1:1,000 dilution; cat. no. ab137550), anti-NADPH-quinone oxidoreductase-1 (NQO1; rabbit polyclonal antibody; 1:1,000 dilution; cat. no. ab217302), anti-heme oxygenase (HO)-1 (rabbit monoclonal antibody; 1:1,500 dilution; cat. no. ab189491), anti-inducible nitric oxide synthase (iNOS; mouse monoclonal antibody; 1:2,000 dilution; cat. no. ab49999), anti-nuclear factor (NF)-кB (rabbit polyclonal antibody; 1:1,000 dilution; cat. no. ab16502) and anti-GAPDH (rabbit monoclonal antibody; 1:10,000 dilution; cat. no. ab181603). All the primary antibodies were obtained from Abcam (Cambridge, MA, USA). Polyclonal horseradish peroxidase-conjugated secondary goat anti-rabbit antibodies $(1: 2,500$; cat. no. sc-2004) and goat anti-mouse antibodies (1:3,500; cat. no. sc-2005) were purchased from Santa Cruz Biotechnology, Inc (Dallas, TX, USA). The relative optical density of the bands was analyzed using ImageJ $2 x$ software (National Institutes of Health).

Biochemical index determination. The biochemical indices of spinal cord samples were determined using commercial kits, following the manufacturers' instructions. The levels of tumor necrosis factor (TNF)- $\alpha$ (cat. no. RAB0480), interleukin (IL)-1 $\beta$ (cat. no. RAB0278) and IL-6 (cat. no. RAB0312) were detected using ELISA kits (Sigma Aldrich; Merck KGaA). The production of nitric oxide (NO) was determined using a $\mathrm{NO}$ assay kit (cat. no. S0024; Beyotime Institute of Biotechnology). Caspase-3 (cat. no. E13183) and -9 (cat. no. KHZ0101) activity levels in the spinal tissue were evaluated using colorimetric protease activation kits (Invitrogen; Thermo Fisher Scientific, Inc.). Commercially available kits for the determination of superoxide dismutase (SOD; cat. no. A001-1) activity, glutathione (GSH; cat. no. A006-2) content, reactive oxygen species (ROS; cat. no. E004-1-1) generation, and malondialdehyde (MDA; cat. no. A003-1) level were purchased from Nanjing Jiancheng Bioengineering Institute.

Statistical analysis. All data are presented as the mean \pm standard error of the mean from at least five independent experiments and were analyzed using SPSS v.17.0 (SPSS, Inc.). One-way analysis of variance with the Student-Newman-Keuls post hoc test was used for comparisons among multiple groups. $\mathrm{P}<0.05$ was considered to indicate statistically significant differences.

\section{Results}

Effect of icariin on locomotor function recovery and spinal cord water content following SCI. The BBB locomotor rating scale was applied to assess the locomotor performance of rats at $0,1,3,7,14$ and 28 days post-injury. As shown in Fig. 1A, 
A
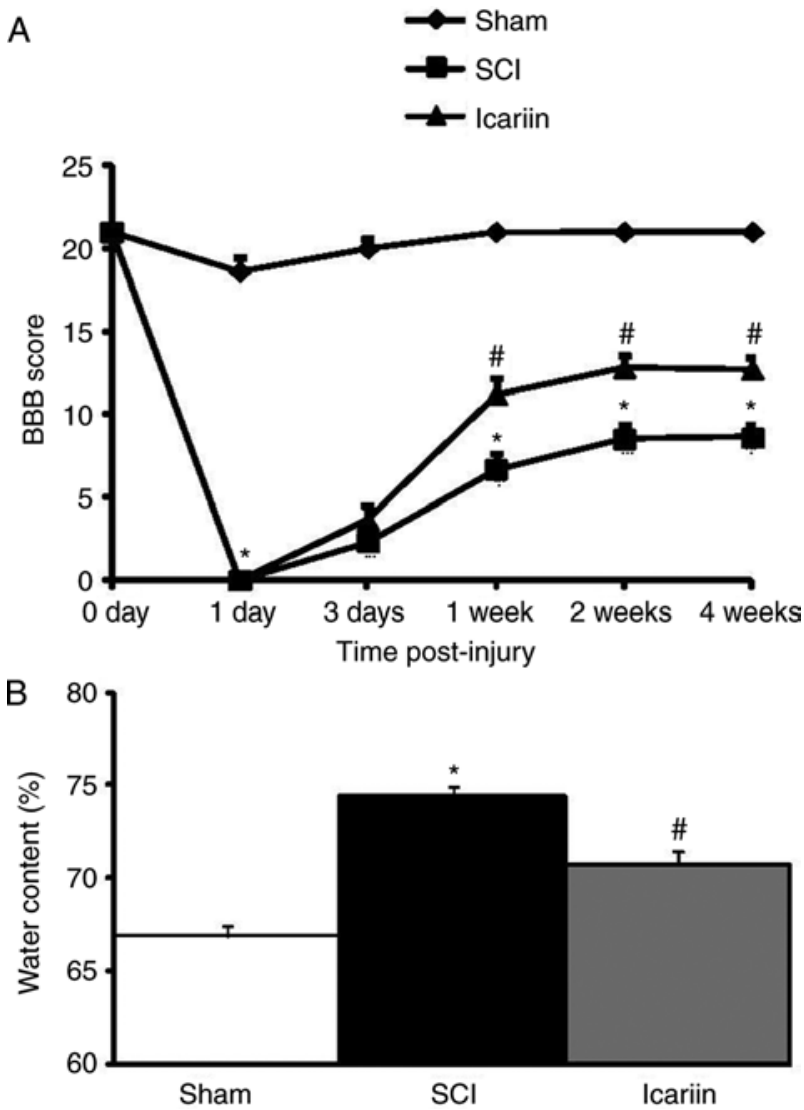

Figure 1. Icariin promotes rat locomotor function recovery and decreases spinal cord water content following SCI. (A) BBB scores of SCI rats with or without application of icariin. (B) Spinal cord water content analysis following 7-day icariin treatment. Data are presented as mean \pm standard error of the mean ( $\mathrm{n}=8$ rats/group). ${ }^{*} \mathrm{P}<0.05$ vs. respective sham group; ${ }^{\#} \mathrm{P}<0.05$ vs. respective SCI group. SCI, spinal cord injury; BBB, Basso, Beattie and Bresnahan.

a marked reduction in locomotor function was observed on the first day after SCI and, over the following days, a gradual recovery was also observed. Compared with the sham group, a significantly faster improvement of locomotor function was recorded in icariin-treated rats at 7,14 and 28 days after the operation $(\mathrm{P}<0.05)$. Rat spinal cord water content was increased in the SCI group compared with the sham group, while icariin significantly alleviated the increase in spinal cord water content $(\mathrm{P}<0.05$; Fig. 1B).

Effect of icariin on the expression and activity of apoptosis-related proteins in rats with SCI. SCI rats exhibited higher expression levels of pro-apoptotic proteins, including Bax, cleaved caspase 3 and cleaved caspase 9. However, the level of the anti-apoptotic factor Bcl-2 was significantly reduced following SCI. Accordingly, caspase 3 and 9 activities were significantly increased upon SCI. All these changes were notably reversed by the application of icariin $(\mathrm{P}<0.05$; Fig. 2$)$.

Effect of icariin on oxidative stress in rats with SCI. SCI rats exhibited higher levels of oxidative stress when compared with the sham group, as evidenced by an increased ROS level and MDA content, as well as decreased SOD activity and GSH levels. All these indices were restored with icariin treatment $(\mathrm{P}<0.05$; Fig. 3).
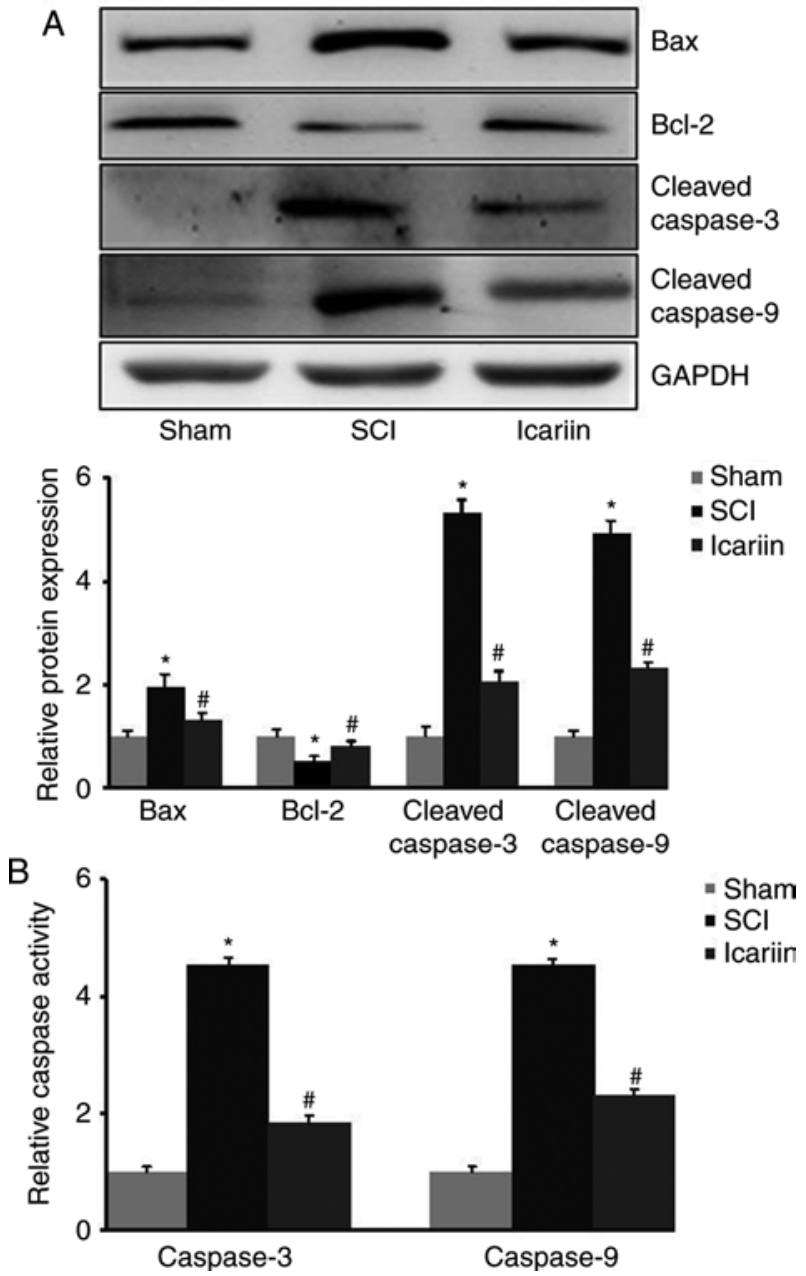

Figure 2. Icariin reduces the apoptosis of spinal cord tissues in SCI rats. (A) Expression of apoptosis-related proteins in SCI rats with or without 7-day application of icariin. (B) Caspase 3 and 9 activities in the rat spinal cord tissue following 7-day icariin treatment. Data are presented as the mean \pm standard error of the mean ( $\mathrm{n}=5$ rats/group). ${ }^{*} \mathrm{P}<0.05$ vs. respective sham group; ${ }^{~} \mathrm{P}<0.05$ vs. respective $\mathrm{SCI}$ group. $\mathrm{SCI}$, spinal cord injury.

Effect of icariin on the expression of inflammation-associated factors. The present study further investigated whether icariin exerted protective effects against SCI damage by inhibiting the expression of inflammatory factors, such as NF- $\mathrm{KB}$ and iNOS. Western blotting revealed a significant $(\mathrm{P}<0.05)$ elevation of $\mathrm{NF}-\kappa \mathrm{B}$ and iNOS protein expression in the SCI group compared with the sham group, whereas icariin treatment significantly decreased the protein expression of NF- $\mathrm{KB}$ and iNOS compared with the SCI group $(\mathrm{P}<0.05$; Fig. 4A). These results indicated that icariin attenuated inflammatory responses in the spinal cord of rats subjected to SCI. In addition, SCI increased the levels of TNF- $\alpha$, IL-1 $\beta$, IL- 6 and NO in comparison to the sham group, and these inflammatory cytokines were reduced in the icariin group compared with the SCI group $(\mathrm{P}<0.05$; Fig. 4B).

To explore the molecular mechanism underlying the neuroprotective effects of icariin administration, western blotting was performed to measure the expression of Nrf2, NQO1 and HO-1, which are all important anti-inflammatory proteins exerting neuroprotective effects during SCI. The results of the western blotting demonstrated that the levels of Nrf2, NQO1 and HO-1 were all elevated following SCI damage, and icariin treatment further enhanced their expression $(\mathrm{P}<0.05 ;$ Fig. $4 \mathrm{C})$. 

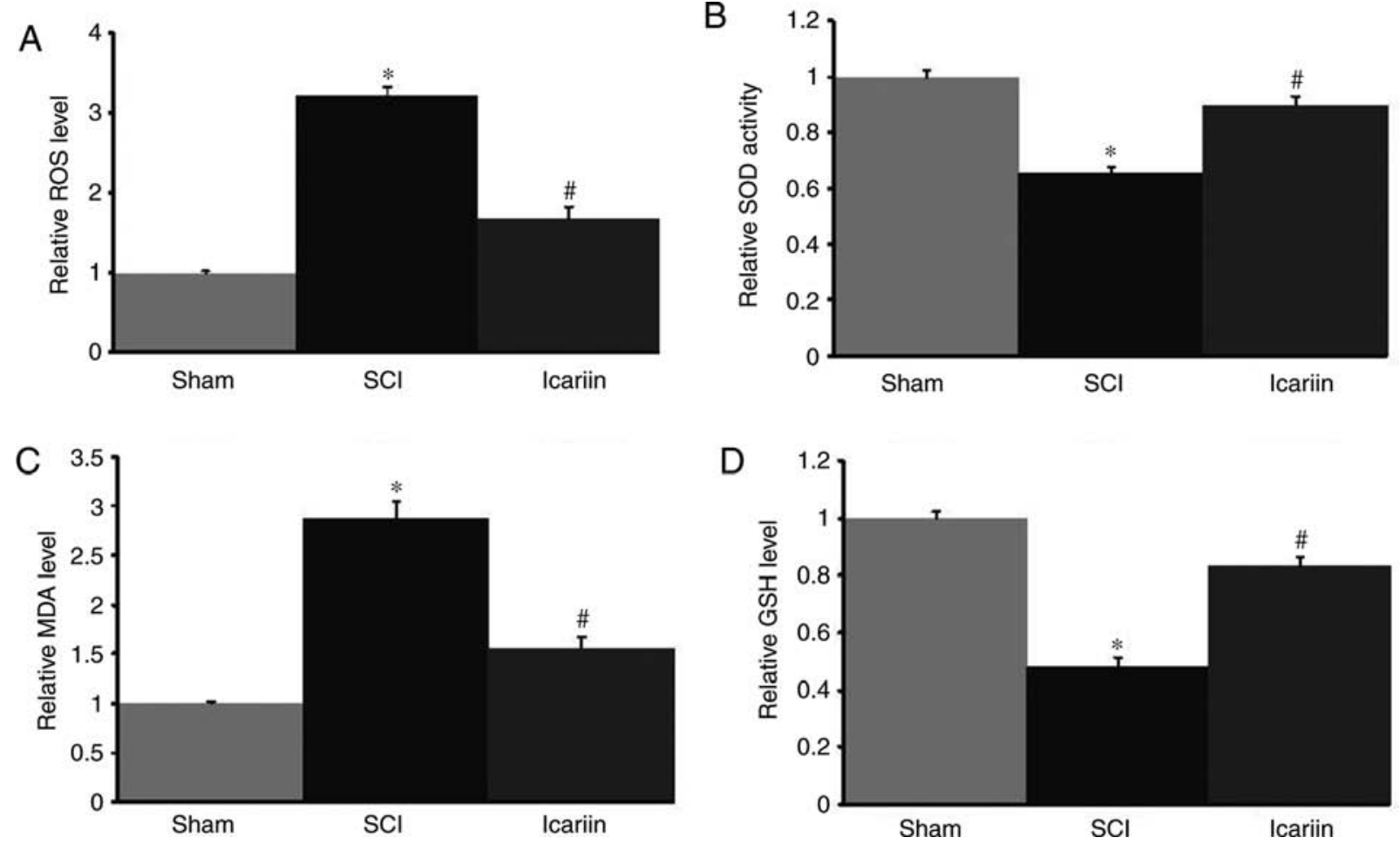

Figure 3. Icariin reduces oxidative stress in the spinal cords of SCI rats. (A) ROS level, (B) SOD activity, (C) MDA level and (D) GSH level in the spinal cord tissues of SCI rats with or without 7-day application of icariin. Data are presented as the mean \pm standard error of the mean ( $\mathrm{n}=5$ rats/group). ${ }^{*} \mathrm{P}<0.05$ vs. respective sham group; ${ }^{\prime} \mathrm{P}<0.05$ vs. respective SCI group. SCI, spinal cord injury; ROS, reactive oxygen species; SOD, superoxide dismutase; GSH, glutathione; MDA, malondialdehyde.

\section{Discussion}

Icariin is a flavonoid found in the Chinese medicinal herb Epimedium sagittatum Maxim. Accumulating evidence indicates that icariin has potent antioxidant, anti-aging, anti-tumor, anti-osteoporosis and neuroprotective properties (16-19). Regarding the neuroprotective effects of icariin, evidence has shown that icariin may protect against Alzheimer's disease $(20,21)$, Parkinson's disease $(21,22)$, multiple sclerosis (21) and SCI (12). In accordance with previously published results, the present study demonstrated that icariin improved the locomotor function of SCI rats, accompanied by a decrease in the spinal cord water content. Furthermore, the present results also revealed that apoptotic-, redox- and inflammatory-related signaling mechanisms were involved in the icariin-mediated anti-SCI effects.

Following SCI, apoptotic death of neural cells is commonly observed in animal models as well as human tissue, and plays an important role in functional disability (23). This process is regulated by Bcl-2, Bax and caspases 3 and 9, indicating the participation of the intrinsic mitochondrial apoptotic pathway in the pathological process following SCI $(24,25)$. In accordance with previous studies, the present study demonstrated that, following SCI, the expression of Bax, cleaved caspase 3 and cleaved caspase 9 , and the activity of caspases 3 and 9 , were all upregulated, accompanied by a decreased Bcl-2 level. Notably, the present data demonstrated that icariin treatment significantly attenuated neural cell apoptosis in SCI rats, as evidenced by the reversal of these apoptosis-related indices. In fact, considerable evidence has suggested the protective role of icariin against mitochondrial apoptotic pathway induction in several pathological conditions (26-28). Due to the absence of morphological results, the present study did not elucidate which types of cells are implicated in the neuroprotective effects of icariin. A recent study by Li et al (29) reported that the neuroprotective effects of icariin may be associated with both neuronal and non-neuronal cells; however, this hypothesis requires further confirmation.

The imbalance between oxidants and antioxidants causes oxidative stress, further resulting in cellular damage. Accumulating evidence has confirmed the critical role of oxidative stress in secondary SCI (30). GSH and SOD are important antioxidants, and MDA is an end-product of lipid peroxidation; these are all widely used as markers of oxidative stress following SCI (31). In the present study, SCI induced marked oxidative stress, as evidenced by increased ROS and MDA levels, with decreased GSH levels and SOD activity, consistent with previous findings. Recently, an increasing number of studies have reported the antioxidant properties of icariin $(32,33)$. Consistently, the present data demonstrated that icariin treatment markedly alleviated oxidative stress responses in the spinal cord tissue of SCI rats, which may represent an important neuroprotective mechanism of this flavonoid.

Inflammation is a crucial factor in secondary damage following SCI, and anti-inflammatory therapy contributes to recovery from SCI $(34,35)$. Nrf2 is a factor that regulates the transcription of cytoprotective genes, including HO-1 and NQO1, thereby playing a pivotal role in anti-inflammatory response (36). Previous studies have confirmed that a number of agents or treatments exert their anti-neuroinflammatory and antioxidant effects in neuronal and neurodegenerative 
A

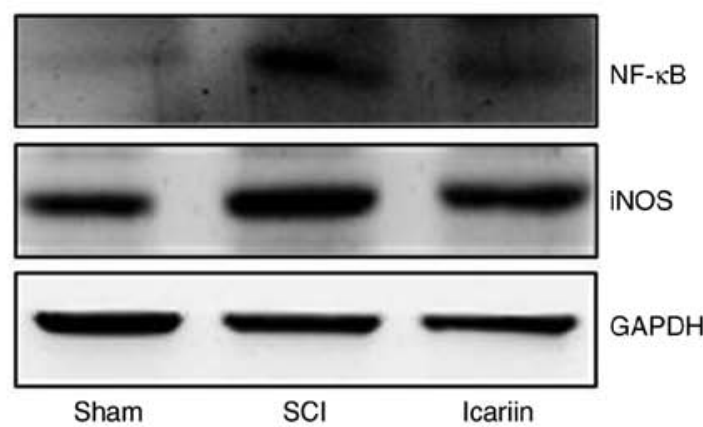

B

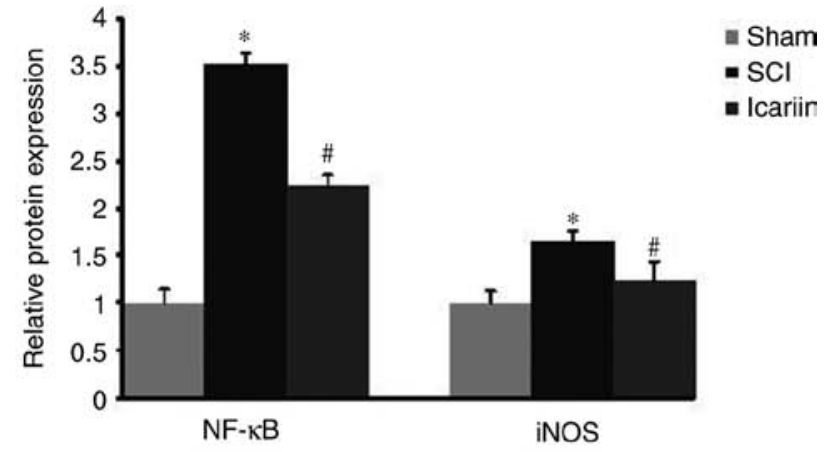

- SCl

Icariin
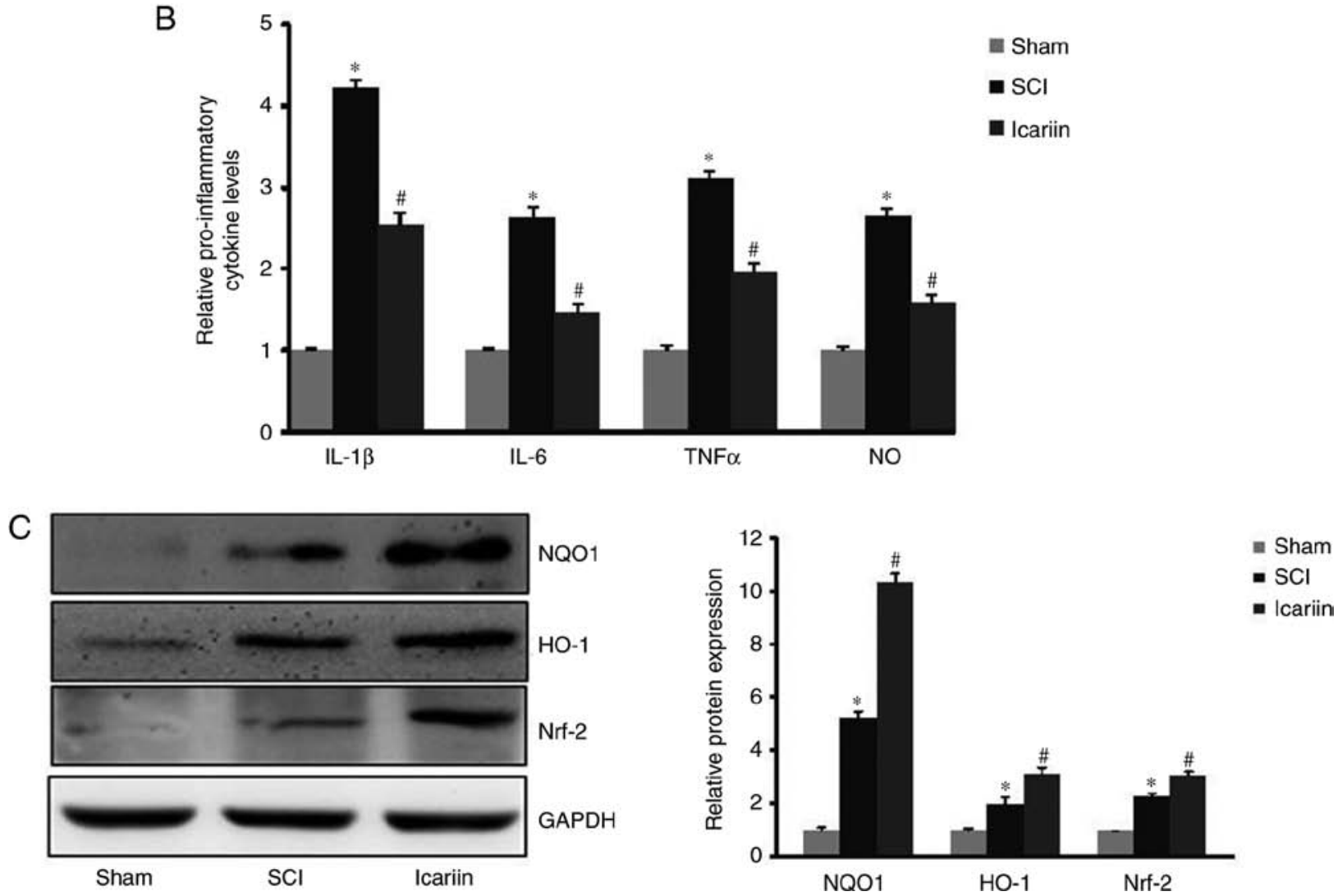

Figure 4. Icariin exerts anti-inflammatory effects in the spinal cords of SCI rats. (A) Expression of NF-kB and iNOS in spinal cord tissues of SCI rats with or without 7-day application of icariin. (B) Analysis of pro-inflammatory cytokine levels of IL-1 $\beta$, IL-6, TNF- $\alpha$ and NO following 7-day icariin treatment. (C) Analysis of anti-inflammatory proteins NQO1, HO-1 and Nrf2 following 7-day icariin treatment. Data are presented as mean \pm standard error of the mean

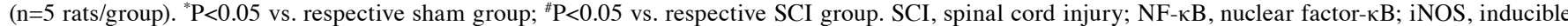
nitric oxide synthase; IL, interleukin; TNF, tumor necrosis factor; NO, nitric oxide; NQO1, NADPH-quinone oxidoreductase-1; HO, heme oxygenase; Nrf2, nuclear factor erythroid 2-related factor 2.

conditions, including SCI, through upregulation of the expression of these signaling molecules (36-39). It was previously demonstrated that icariin exhibited potent anti-inflammatory activity $(16,40,41)$. The present study revealed that the expression of all three anti-inflammatory molecules was upregulated in SCI rats, and icariin treatment further promoted their expression. These findings indicated that there may be a compensatory or adaptive upregulation of anti-inflammatory molecules in SCI, which may help alleviate SCI-induced damage, and icariin treatment may further enhance this protective effect.

$\mathrm{NF}-\kappa \mathrm{B}$ is a transcription factor that controls the gene expression of a number of inflammation-related proteins that contribute to SCI, such as TNF- $\alpha$, IL-1 $\beta$ and IL-6 (42). It has been documented that icariin and its metabolite, icariside II, exert their anti-inflammatory effects via inhibition of the NF- $\mathrm{KB}$ signaling pathway (43). In the present study, a significant elevation of NF-kB p65 subunit expression was observed in SCI rats, and icariin treatment markedly suppressed its expression, implicating the involvement of NF- $\mathrm{KB}$ pathway inhibition in icariin-mediated anti-inflammatory action during the attenuation of SCI in rats.

It was reported that inflammatory reactions may cause increased expression of iNOS, leading to the excessive NO production post-SCI $(9,44)$. High levels of NO exert a cytotoxic effect on the spinal cord. In fact, selective inhibition of iNOS by aminoguanidine has been found to promote the recovery of neurological function in rats subjected 
to SCI (45). Consistent with previous results, the present findings demonstrated that the protein level of iNOS and NO production were evidently increased in SCI rats. More importantly, the administration of icariin markedly reduced these indices in the traumatic SCI rat model. Thus, it may be concluded that icariin exerts its protective effects against spinal cord functional impairment by suppressing iNOS/NO. Although the present study and a recent report (29) support the possible involvement of the suppression of iNOS/NO signaling in icariin-mediated neuroprotection against SCI, the underlying mechanism remains unclear and requires further investigation.

Previous studies have shown that icariin possesses anti-apoptotic, antioxidant and anti-inflammatory bioactivities, thereby improving the recovery of locomotor function in SCI rats $(12,29,46)$. The findings of the present study further supported the soundness of these arguments by examining additional apoptotic-, redox- and inflammatory-related mechanisms. The results of the present study included the attenuating effect of icariin on the activities of caspases 3 and 9 and levels of NF- $\mathrm{KB}$, IL-6, NO and ROS, as well as the enhancement of anti-inflammatory factors, such as NQO-1, HO-1 and Nrf-2. It should be noted that, although the present study using an animal model demonstrated that icariin treatment may be a promising therapeutic strategy for SCI, the therapeutic effectiveness of this drug requires further confirmation in clinical trials.

\section{Acknowledgements}

Not applicable.

\section{Funding}

The present study was supported by President Foundation, Aohongboze Foundation of Liaoning Medical University (grant no. XZJJ20140230), and partially by National Natural Science Foundation of China (grant no. 81700519).

\section{Availability of data and materials}

The datasets generated and analyzed in the present study are available from the corresponding author on reasonable request.

\section{Authors' contributions}

HD conceived and designed the study. GJ performed the experiments and completed the manuscript draft. GJ, YZ and WL analyzed the data. All the authors have read and approved the final version of this manuscript for publication.

\section{Ethics approval and consent to participate}

All procedures were approved by the Experimental Animal Care and Use Committee at Jinzhou Medical University.

\section{Patient consent for publication}

Not applicable.

\section{Competing interests}

The authors declare that they have no competing interests.

\section{References}

1. Xu M, Li H, Zhao Z, Yang Y, Sun Z, Han H, Zhang X and Reinhardt JD: Environmental barriers, functioning and quality of life in 2008 Wenchuan earthquake victims with spinal cord injury eight years after the disaster: A cross-sectional study. J Rehabil Med 50: 866-871, 2018.

2. Maitan P, Frigerio S, Conti A, Clari M, Vellone E and Alvaro R: The effect of the burden of caregiving for people with spinal cord injury (SCI): A cross-sectional study. Ann Ist Super Sanita 54: 185-193, 2018.

3. Quadri SA, Farooqui M, Ikram A, Zafar A, Khan MA, Suriya SS, Claus CF, Fiani B, Rahman M, Ramachandran A, et al: Recent update on basic mechanisms of spinal cord injury. Neurosurg Rev: July 11, 2018 (Epub ahead of print).

4. Sobrido-Camean D and Barreiro-Iglesias A: Role of Caspase- 8 and fas in cell death after spinal cord injury. Front Mol Neurosci 11: 101, 2018.

5. Rudman MD, Choi JS, Lee HE, Tan SK, Ayad NG and Lee JK: Bromodomain and extraterminal domain-containing protein inhibition attenuates acute inflammation after spinal cord injury. Exp Neurol 309: 181-192, 2018.

6. Donovan J and Kirshblum S: Clinical trials in traumatic spinal cord injury. Neurotherapeutics 15: 654-668, 2018.

7. Jiang Y, Gong FL, Zhao GB and Li J: Chrysin suppressed inflammatory responses and the inducible nitric oxide synthase pathway after spinal cord injury in rats. Int J Mol Sci 15: 12270-12279, 2014.

8. Kang S, Liu S, Li H, Wang D and Qi X: Baicalin effects on rats with spinal cord injury by anti-inflammatory and regulating the serum metabolic disorder. J Cell Biochem 119: 7767-7779, 2018.

9. Zhou L, Ouyang L, Lin S, Chen S, Liu Y, Zhou W and Wang X: Protective role of $\beta$-carotene against oxidative stress and neuroinflammation in a rat model of spinal cord injury. Int Immunopharmacol 61: 92-99, 2018.

10. Liu P, Zhang Z, Wang Q, Guo R and Mei W: Lithium chloride facilitates autophagy following spinal cord injury via ERK-dependent pathway. Neurotox Res 32: 535-543, 2017.

11. Silva NA, Sousa N, Reis RL and Salgado AJ: From basics to clinical: A comprehensive review on spinal cord injury. Prog Neurobiol 114: 25-57, 2014.

12. Tohda $C$ and Nagata A: Epimedium koreanum extract and its constituent icariin improve motor dysfunction in spinal cord injury. Evid Based Complement Alternat Med 2012: 731208, 2012 .

13. Wang C, Liu C, Gao K, Zhao H, Zhou Z, Shen Z, Guo Y, Li Z, Yao T and Mei X: Metformin preconditioning provide neuroprotection through enhancement of autophagy and suppression of inflammation and apoptosis after spinal cord injury. Biochem Biophys Res Commun 477: 534-540, 2016.

14. Yacoub A, Hajec MC, Stanger R, Wan W, Young H and Mathern BE: Neuroprotective effects of perflurocarbon (oxycyte) after contusive spinal cord injury. J Neurotrauma 31: 256-267, 2014.

15. Dai H, Song D, Xu J,Li B, Hertz L and Peng L: Ammonia-induced Na,K-ATPase/ouabain-mediated EGF receptor transactivation, MAPK/ERK and PI3K/AKT signaling and ROS formation cause astrocyte swelling. Neurochem Int 63: 610-625, 2013.

16. Wang GQ, Li DD, Huang C, Lu DS, Zhang C, Zhou SY, Liu J and Zhang F: Icariin reduces dopaminergic neuronal loss and microglia-mediated inflammation in vivo and in vitro. Front Mol Neurosci 10: 441, 2018.

17. Zhou J, Wu J, Chen X, Fortenbery N, Eksioglu E, Kodumudi KN, Pk EB, Dong J, Djeu JY and Wei S: Icariin and its derivative, ICT, exert anti-inflammatory, anti-tumor effects, and modulate myeloid derived suppressive cells (MDSCs) functions. Int Immunopharmacol 11: 890-898, 2011.

18. Liu Y, Zuo H, Liu X, Xiong J and Pei X: The antiosteoporosis effect of icariin in ovariectomized rats: A systematic review and meta-analysis. Cell Mol Biol (Noisy-le-grand) 63: 124-131, 2017.

19. Li C, Li Q, Mei Q and Lu T: Pharmacological effects and pharmacokinetic properties of icariin, the major bioactive component in Herba Epimedii. Life Sci 126: 57-68, 2015. 
20. Sheng C, Xu P, Zhou K, Deng D, Zhang C and Wang Z: Icariin attenuates synaptic and cognitive deficits in an A $\beta 1$-42-induced rat model of Alzheimer's disease. Biomed Res Int 2017: 7464872, 2017.

21. Jin J, Wang H, Hua X, Chen D, Huang C and Chen Z: An outline for the pharmacological effect of icariin in the nervous system. Eur J Pharmacol 842: 20-32, 2019.

22. Chen WF, Wu L, Du ZR, Chen L, Xu AL, Chen XH, Teng JJ and Wong MS: Neuroprotective properties of icariin in MPTP-induced mouse model of Parkinson's disease: Involvement of PI3K/Akt and MEK/ERK signaling pathways. Phytomedicine 25: 93-99, 2017.

23. Chen XB, Wang ZL, Yang QY, Zhao FY, Qin XL, Tang XE, Du JL, Chen ZH, Zhang K and Huang FJ: Diosgenin glucoside protects against spinal cord injury by regulating autophagy and alleviating apoptosis. Int J Mol Sci 19: pii: E2274, 2018.

24. Wang Z, Zhou L, Zheng X, Chen G, Pan R, Li J and Liu W: Autophagy protects against PI3K/Akt/mTOR-mediated apoptosis of spinal cord neurons after mechanical injury. Neurosci Lett 656: 158-164, 2017.

25. Zhao H, Chen S, Gao K, Zhou Z, Wang C, Shen Z, Guo Y, Li Z, Wan Z, Liu C and Mei X: Resveratrol protects against spinal cord injury by activating autophagy and inhibiting apoptosis mediated by the SIRT1/AMPK signaling pathway. Neuroscience 348: 241-251, 2017.

26. Qian ZQ, Wang YW, Li YL, Li YQ, Ling-Zhu and Yang DL: Icariin prevents hypertension-induced cardiomyocyte apoptosis through the mitochondrial apoptotic pathway. Biomed Pharmacother 88: 823-831, 2017.

27. Wang Q, Hao J, Pu J, Zhao L, Lü Z, Hu J, Yu Q, Wang Y, Xie Y and Li G: Icariin induces apoptosis in mouse MLTC-10 Leydig tumor cells through activation of the mitochondrial pathway and down-regulation of the expression of piwil4. Int J Oncol 39: 973-980, 2011

28. Li S, Dong P, Wang J, Zhang J, Gu J, Wu X, Wu W, Fei X, Zhang Z, Wang Y, et al: Icariin, a natural flavonol glycoside, induces apoptosis in human hepatoma SMMC-7721 cells via a ROS/JNK-dependent mitochondrial pathway. Cancer Lett 298: 222-230, 2010

29. Li H, Zhang X, Zhu X, Qi X, Lin K and Cheng L: The effects of icariin on enhancing motor recovery through attenuating pro-inflammatory factors and oxidative stress via mitochondrial apoptotic pathway in the mice model of spinal cord injury. Front Physiol 9: 1617, 2018.

30. Kong G, Huang Z, Ji W, Wang X, Liu J, Wu X, Huang Z, Li R and Zhu Q: The ketone metabolite $\beta$-hydroxybutyrate attenuates oxidative stress in spinal cord injury by suppression of class I histone deacetylases. JJ Neurotrauma 34: 2645-2655, 2017.

31. Cong L and Chen W: Neuroprotective effect of ginsenoside Rd in spinal cord injury rats. Basic Clin Pharmacol Toxicol 119: 193-201, 2016.

32. Sun S, Liu L, Tian X, Guo Y, Cao Y, Mei Y and Wang C: Icariin attenuates high glucose-induced apoptosis, oxidative stress, and inflammation in human umbilical venous endothelial cells. Planta Med 85: 473-482, 2019.
33. Zheng Y, Zhu G, He J, Wang G, Li D and Zhang F: Icariin targets Nrf2 signaling to inhibit microglia-mediated neuroinflammation. Int Immunopharmacol 73: 304-311, 2019.

34. Xu G, Shi D, Zhi Z, Ao R and Yu B: Melatonin ameliorates spinal cord injury by suppressing the activation of inflammasomes in rats. J Cell Biochem 120: 5183-5192, 2019.

35. Li Z, Yao F, Cheng L, Cheng W, Qi L, Yu S, Zhang L, Zha X and Jing J: Low frequency pulsed electromagnetic field promotes the recovery of neurological function after spinal cord injury in rats. J Orthop Res 37: 449-456, 2019.

36. Park SY, Kim YH and Park G: Cucurbitacins attenuate microglial activation and protect from neuroinflammatory injury through Nrf2/ARE activation and STAT/NF- $\mathrm{BB}$ inhibition. Neurosci Lett 609: 129-136, 2015

37. Wei W, Shurui C, Zipeng Z, Hongliang D, Hongyu W, Yuanlong L, Kang Z, Zhaoliang S, Yue G, Chang L and Mei X: Aspirin suppresses neuronal apoptosis, reduces tissue inflammation, and restrains astrocyte activation by activating the Nrf2/HO-1 signaling pathway. Neuroreport 29: 524-531, 2018.

38. Xia P, Gao X, Duan L, Zhang W and Sun YF: Mulberrin (Mul) reduces spinal cord injury (SCI)-induced apoptosis, inflammation and oxidative stress in rats via miroRNA-337 by targeting Nrf-2. Biomed Pharmacother 107: 1480-1487, 2018.

39. Kim Y, Kim J, Ahn M and Shin T: Lithium ameliorates rat spinal cord injury by suppressing glycogen synthase kinase- $3 \beta$ and activating heme oxygenase-1. Anat Cell Biol 50: 207-213, 2017.

40. Kong L, Liang X, Liu A, Yang X, Luo Q, Lv Y and Dong J: Icariin inhibits inflammation via immunomodulation of the cutaneous hypothalamus-pituitary-adrenal axis in vitro. Clin Exp Dermatol 44: 144-152, 2019.

41. Hua W, Zhang Y, Wu X, Kang L, Tu J, Zhao K, Li S, Wang K, Song Y, Luo R, et al: Icariin attenuates interleukin-1 $\beta$-induced inflammatory response in human nucleus pulposus cells. Curr Pharm Des 23: 6071-6078, 2018.

42. Chen S, Ye J, Chen X, Shi J, Wu W, Lin W, Lin W, Li Y, $\mathrm{Fu} \mathrm{H}$ and Li S: Valproic acid attenuates traumatic spinal cord injury-induced inflammation via STAT1 and NF- $\mathrm{B}$ pathway dependent of HDAC3. J Neuroinflammation 15: 150, 2018.

43. Hwang E, Lin P, Ngo HTT, Gao W, Wang YS, Yu HS and Yi TH: Icariin and icaritin recover UVB-induced photoaging by stimulating Nrf2/ARE and reducing AP-1 and NF- $\kappa$ B signaling pathways: A comparative study on UVB-irradiated human keratinocytes. Photochem Photobiol Sci 17: 1396-1408, 2018.

44. Wang S and Ren D: Allicin protects traumatic spinal cord injury through regulating the HSP70/Akt/iNOS pathway in mice. Mol Med Rep 14: 3086-3092, 2016.

45. Li Z, Du J, Sun H, Mang J, He J, Wang J, Liu Hand Xu Z: Effects of the combination of methylprednisolone with aminoguanidine on functional recovery in rats following spinal cord injury. Exp Ther Med 7: 1605-1610, 2014

46. Ren XS, Ding W and Yang XY: Neuroprotective effect of icariin on spinal cord injury in rats. Zhongguo Gu Shang 31: 1054-1060, 2018 (In Chinese) 